\title{
ANÁLISIS ESPACIO-TEMPORAL DE LA MALARIA EN MATINA, LIMÓN, COSTA RICA
}

\author{
SPACE-TIME ANALYSIS OF MALARIA IN MATINA, \\ LIMÓN, COSTA RICA
}

\author{
Silvia Argüello Vargas ${ }^{1}$ \\ Elba de la Cruz Malavassi ${ }^{2}$ \\ Marco V. Herrero Acosta ${ }^{3}$ \\ Regional de Estudios en Sustancias Tóxicas, \\ Universidad Nacional, Costa Rica
}

\section{RESUMEN}

El objetivo de este estudio fue establecer el patrón espacio-temporal de la malaria en Matina y relacionarlo con factores ambientales. Se utilizaron tecnologías espaciales para capturar, almacenar, analizar y visualizar información relacionada con localidades y viviendas. Los atributos no espaciales fueron analizados usando pruebas paramétricas y no paramétricas. Los datos fueron obtenidos de las bases de datos de casos clínicos del Área Rectora del Ministerio de Salud en Matina. Se presentan los descriptores puntuales de las localidades positivas para los años 2005 y 2006 y en los grupos de viviendas positivo y negativo en la localidad piloto. Se propone una clasificación de áreas macroambientales en el cantón y se relaciona con la distribución de la

1 Máster.Instituto Regional de Estudios en Sustancias Tóxicas (IRET), Facultad de Ciencias de la Tierra y el Mar, Universidad Nacional. Apartado 86-3000. Heredia, Costa Rica. Correo electrónico: sarguell@una.cr

2 Doctora. Instituto Regional de Estudios en Sustancias Tóxicas (IRET), Facultad de Ciencias de la Tierra y el Mar, Universidad Nacional. Apartado 86-3000. Heredia, Costa Rica. Correo electrónico: elbamd@ gmail.com

3 Doctor. Facultad de Ciencias de la Tierra y el Mar, Universidad Nacional. Apartado 86-3000. Heredia, Costa Rica. Correo electrónico: herrero1958@hotmail.com

Fecha de recepción: 18 de diciembre de 2014

Fecha de aceptación: 9 de marzo de 2015 
Incidencia Parasitaria Anual (IPA). Se identificaron factores de riesgo a nivel de vivienda en la localidad piloto. Se describe la ocurrencia temporal de la actividad malárica en el cantón. El patrón espacio-temporal que se presenta en este informe puede servir de línea base para estudiar cambios que podrían ocurrir en el futuro.

Palabras clave: Plasmodium spp., malaria, Costa Rica, Región Huetar Atlántica, SIG

\begin{abstract}
The purpose of this study was to describe the space-time pattern of the disease, and relate it to environmental factors. Spatial technologies were used to collect, store, analyze and display information regarding locations and household locations. Non-spatial attributes were analyzed using parametric and non parametric tests. The information was obtained from databases of clinical cases form the Governing Area of the Health Ministry in Matina. Centrographic parameters were calculated for localities within Matina and for households within the pilot location. Parasitic Incidence (IPA) was associated with a proposed environmental classification for Matina. At the household level, risk factors were determined. The temporal pattern of the disease in Matina is described. A similar temporal trend is shown for households within the pilot location. This is the first time that the information collected in the Matina Governing Area is used to describe the spatial patterns of malaria. This pattern will be useful as a comparative baseline for future studies.
\end{abstract}

Keywords: Plasmodium spp., malaria, Costa Rica, Región Huetar Atlántica, GIS

\title{
Introducción
}

La malaria es una enfermedad de relevancia en salud pública. En lugares propicios para que se establezca su ciclo natural es transmitida por mosquitos desde un huésped infectado a uno susceptible. En Costa Rica los casos pueden ser importados (se originan en otros países y son detectados en la población inmigrante) o autóctonos (se transmiten in situ en las áreas endémicas). Se sospecha que el vector es Anopheles albimanus y el agente etiológico presente en la mayor parte de los casos es Plasmodium vivax. Sin embargo, en el 2006 el Ministerio de Salud declaró alerta nacional a partir del hallazgo de infecciones mixtas $P$. vivax- $P$. falciparum en el cantón de Matina.

En el país, la enfermedad es endémica en la región Huetar Atlántica Sur. Durante el periodo de estudio el $90 \%$ de los casos ocurrió en el cantón de Matina, presentando una incidencia parasitaria anual (IPA) de 76.95 en el 2005 y de 69.11 en el 2006.

El trabajo aquí presentado se desarrolló como parte del "Programa Regional de Acción y Demostración de Alternativas Sostenibles para el Control de Vectores de la Malaria sin uso de DDT en México y América Central", el cual implementó un sistema de información geográfica en el 
Área Rectora de Salud de Matina para realizar un análisis sobre la situación de la malaria en el cantón de Matina.

Es esperable que el trabajo que se presenta sirva de base para el manejo sistemático de la información, así como para el análisis espacial de la enfermedad y su seguimiento, coadyuvando en la toma de decisiones relativas a la epidemiología de la enfermedad. Asimismo, podría servir como modelo para el estudio de otras enfermedades.

\section{Materiales y Métodos}

\section{Sitio de estudio}

El estudio se llevó a cabo en dos niveles geográficos, el primero a nivel de localidades del cantón de Matina y el segundo a nivel de viviendas en la localidad piloto (Estrada).

\section{Recolección de la información primaria}

El Área Rectora de Salud Matina suministró la información para la generación de las bases de datos. La información contenía los datos de los casos anuales de malaria para el periodo 2005 - 2006, las coordenadas geográficas de las viviendas obtenidas con un GPS in situ, el número de las viviendas, los datos generales de las localidades y la encuesta de línea basal, esta incluyó aspectos relacionados con la población que habita la casa, el estado socioeconómico de la familia, la estructura de la vivienda, otras características de la vivienda y la tenencia de animales.

\section{Análisis espacial}

El análisis espacial estuvo comprendido por el cálculo de los descriptores puntuales, la caracterización ambiental de las localidades, una clasificación ambiental y una evaluación epidemiológica. A continuación, se describe la metodología de cada componente según el nivel geográfico.

\section{Descriptores puntuales}

Nivel geográfico de localidades: Se seleccionaron todas las localidades del cantón de Matina que tenían datos sobre número de casos por año, población estimada e IPA. Se crearon dos archivos primarios, uno para el 2005 y otro para el 2006. 
Se calculó el centro promedio, la distancia estándar y la elipse desviacional estándar usando CrimeStat. Para el cálculo del centro promedio se usó la incidencia porcentual por localidad como peso estadístico. Los centros promedios fueron comparados entre años haciendo uso de una prueba de t para grupos no pareados.

Nivel geográfico de Vivienda: Se incluyeron para este análisis aquellas viviendas a las que se les aplicó la encuesta de línea basal, y de las cuales había sido posible obtener el número de habitantes por vivienda. Se crearon dos archivos primarios para el año 2006, uno para el grupo positivo (con al menos un caso de malaria confirmado por labortorio) y otro para el grupo negativo (sin reporte de casos de malaria). Haciendo uso de CrimeStat III se calcularon el centro promedio, la distancia estándar y la elipse desviacional estándar. Para el cálculo del centro promedio, se usó la incidencia porcentual por vivienda como peso estadístico. Estos parámetros fueron desplegados en un polígono de referencia para el área de Estrada. Los centros promedios fueron comparados entre años haciendo uso de una prueba de $t$ para grupos no pareados.

\section{Caracterización de las localidades}

Se utilizó la extensión geoprocesadora de ArcView para asignar características macroambientales a cada una de las localidades de Matina. El cuadro 1 representa para cada variable macroambiental las diferentes categorías existentes en el cantón de Matina; no en todas estas se presentaron localidades positivas. 
Cuadro 1. Categorías, códigos y escala de las variables para la caracterización de Matina.

\begin{tabular}{|c|c|c|c|}
\hline Variable & Categorías existentes para Matina & Código & Escala \\
\hline Meses secos & 1 mes seco durante el año. & $(1.1)$ & 1:200000 \\
\hline Cobertura 2000 & $\begin{array}{l}\text { Cobertura forestal } \\
\text { Deforestación } \\
\text { No forestal }\end{array}$ & $\begin{array}{l}(2.1) \\
(2.2) \\
(2.3)\end{array}$ & $1: 50000$ \\
\hline Geología & $\begin{array}{l}\text { Depósitos aluviales y coluviales } \\
\text { Rocas sedimentarias vocanoclásticas } \\
\text { Rocas sedimentarias de aguas profundas } \\
\text { Rocas volcánicas }\end{array}$ & $\begin{array}{l}(3.1) \\
(3.2) \\
(3.3) \\
(3.4)\end{array}$ & $1: 50000$ \\
\hline $\begin{array}{l}\text { Características del } \\
\text { terreno }\end{array}$ & $\begin{array}{l}\text { Plano (pendiente de } 0 \text { a } 2 \% \text { ) } \\
\text { Suavemente ondulado (pendiente } 2 \text { a } 15 \% \text { ) } \\
\text { Moderamente ondulado (pendiente } 15 \text { a } 30 \% \text { ) } \\
\text { Fuertemente ondulado (pendiente } 30 \text { a } 60 \% \text { ) } \\
\text { Escarpado (pendiente }>60 \% \text { ) }\end{array}$ & $\begin{array}{l}(4.1) \\
(4.2) \\
(4.3) \\
(4.4) \\
(4.5)\end{array}$ & 1: 200000 \\
\hline Elevación msnm & $\begin{array}{l}0 \\
100 \\
>100 \\
\end{array}$ & $\begin{array}{l}(5.1) \\
(5.2) \\
(5.3)\end{array}$ & $1: 200000$ \\
\hline $\begin{array}{l}\text { Brillo Solar (horas } \\
\text { de insolación } \\
\text { solar por día) }\end{array}$ & $\begin{array}{l}0 \\
3 \\
4 \\
5\end{array}$ & $\begin{array}{l}(6.1) \\
(6.2) \\
(6.3) \\
(6.4)\end{array}$ & $1: 200000$ \\
\hline Precipitación mm & $\begin{array}{l}3500 \\
4000 \\
4500 \\
5000\end{array}$ & $\begin{array}{l}(7.1) \\
(7.2) \\
(7.3) \\
(7.4)\end{array}$ & 1:200000 \\
\hline Tipo de Suelo & $\begin{array}{l}\text { Eop } \\
\text { Epp } \\
\text { Hsp } \\
\text { Le p } \\
\text { Leso } \\
\text { Lmp } \\
\text { Ltp } \\
\text { Wm mo } \\
\text { Ut e } \\
\text { Ut fo } \\
\text { Ut mo }\end{array}$ & $\begin{array}{l}(8.1) \\
(8.2) \\
(8.3) \\
(8.4) \\
(8.5) \\
(8.6) \\
(8.7) \\
(8.8) \\
(8.9) \\
(8.10) \\
(8.11)\end{array}$ & $1: 200000$ \\
\hline
\end{tabular}




\section{Clasificación ambiental}

Nivel geográfico de localidades: Todas las áreas del cantón de Matina que tenían al menos una localidad positiva se clasificaron según los factores ambientales (cuadro 1), tanto para 2005 como para 2006. Se usó un dendrograma para mostrar la diferenciación de las áreas.

Nivel geográfico de Vivienda: Para el análisis macroambiental se construyó un polígono de referencia para la localidad de Estrada, definido por las siguientes coordenadas en Lambert Norte 616142.21129 $\mathrm{O}, 227980.58295 \mathrm{~N}$ (esquina superior derecha) y $614012.18988 \mathrm{O}$, $226138.74765 \mathrm{~N}$ (esquina inferior izquierda). Este polígono se intersectó con el mapa temático de Matina desplegado por grupos macroambientales. Para la caracterización de cuerpos de agua permanentes, se ubicaron los ríos utilizando la capa de ríos 1:50000 del Atlas Digital de Costa Rica (Ortiz, 2004) sobre un mapa del cantón de Matina y se ubicaron los puntos representando las localidades del 2005 y del 2006. Se creó una zona de amortiguamiento a partir de la capa de ríos con un radio de $2 \mathrm{~km}$. Finalmente se contaron todas las localidades que se encuentran dentro de la zona de amortiguamiento. Los datos recolectados en la encuesta se compararon entre el grupo negativo y el positivo. Para variables numéricas, se utilizó la prueba de Mann-Whitney. Para variables categóricas, se utilizó una prueba de comparación de dos proporciones para grupos independientes y se calculó el RR (riesgo relativo) entre grupos y la frecuencia observada cuando las diferencias observadas fueron significativas.

\section{Evaluación epidemiológica}

Nivel geográfico de localidades (factores macroambientale): Se calculó el IPA promedio por área macroambiental. Para cada una de estas áreas, se hizo el cálculo de RR (riesgo relativo) y para los factores con riesgo $>1$ se calculó \%RAP (porcentaje de riesgo atribuible poblacional) por año.

(Nivel geográfico de Vivienda. (factores microambientales): Se calculó el \%RAP para los factores de riesgo identificados.

\section{Análisis de tendencias temporales}

Nivel geográfico de localidades: Se graficaron dos variables, el número de casos por mes y el número de localidades positivas, a partir del tiempo inicial de observación en el 2005 y hasta el último mes del 2006. Para ambas 
variables se calculó el promedio móvil y se realizó un ANDEVA. Se graficó la variación de los promedios de la latitud y de la longitud para los 24 meses de estudio. Se calculó el intervalo de confianza de $95 \%$ y el coeficiente de variación para el promedio de los promedios de cada variable.

Nivel geográfico de Vivienda: Se graficaron dos variables, el número de casos y el número de viviendas positivas, a partir del tiempo inicial de observación y hasta su finalización en el 2006. Para ambas variables, se calculó el promedio móvil y se aplicó un ANDEVA. Se graficó la variación mensual en latitud y en longitud durante el periodo de observación. Se calculó el coeficiente de variación y el intervalo de confianza de 95\% para el promedio de los promedios de la longitud y de la latitud de las viviendas positivas.

\section{Resultados}

En el año 2005 ocurrieron 2935 casos de malaria en 45 localidades del cantón de Matina, en las cuales había una población a riesgo de 38139 habitantes que generó un IPA de 76.95 casos por mil habitantes. En el año 2006 ocurrieron 2608 casos de malaria en las mismas localidades, con excepción de 2, para un total de 37706 habitantes lo cual generó un IPA de 69.11 casos por mil habitantes. Las figuras 1 y 2 muestran, respectivamente, el centro promedio, la distancia estándar y la elipse desviacional estándar para las localidades y para las viviendas de la localidad piloto en el cantón de Matina. En la figura 1 se muestra la distribución del IPA mediante un mapa de símbolo graduado mientras que la figura 2 representa la distribución de las viviendas positivas mediante una clasificación de símbolo graduado por número de casos. 
Figura 1. Centro promedio, distancia estándar y elipse desviacional estándar para las localidades en el cantón de Matina.
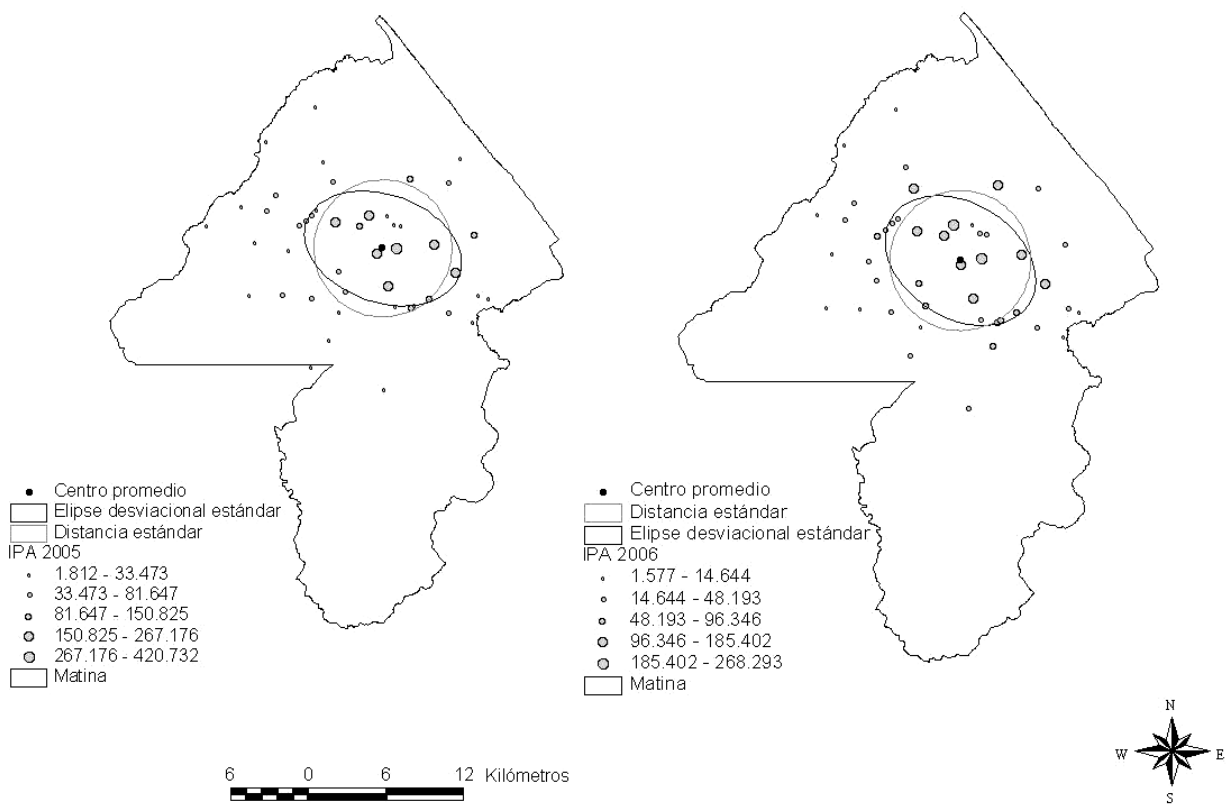
Figura 2. Centro promedio, distancia estándar y elipse desviacional estándar de los grupos, positivo y negativo, de viviendas en la localidad de Estrada, Matina.
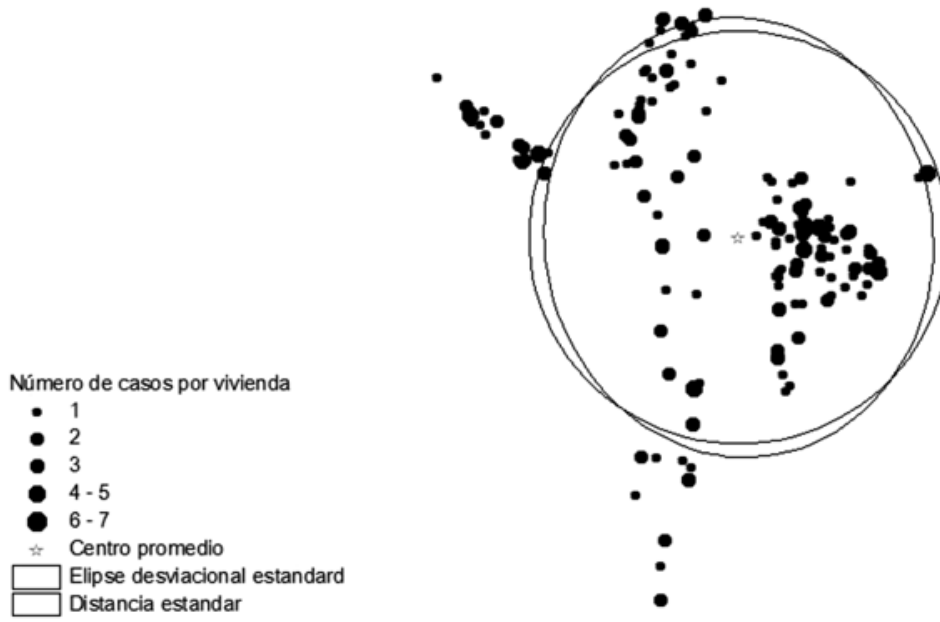

0.6

En cuanto a las localidades, el centro promedio para el año 2005 fue de $615654.80549 \mathrm{O}, 227410.83016 \mathrm{~N}$ y para el año 2006 fue de $615104.11493 \mathrm{O}, 227302.80548 \mathrm{~N}$. No se observaron diferencias en estos parámetros cuando se compararon entre años a nivel de localidad. El valor de $p$ para la longitud fue de 0.9561 y para la latitud fue de 0.6939 .

Para las viviendas, el centro promedio del grupo positivo fue $615325.90420 \mathrm{O}, 227195.15019 \mathrm{~N}$ y para el negativo fue de 615292.86739 O, 227240.38904 N. Tampoco se hallaron diferencias cuando se compararon estos descriptores entre el grupo positivo y el grupo negativo de viviendas a nivel de la localidad de Estrada. Para la longitud el valor de $p$ fue de 0.2734 y para la latitud fue de 0.1540 . 
Las figuras 3 y 4 muestran las áreas macroambientales del cantón de Matina y su distribución; estas áreas se basan en las características mostradas en el cuadro 1. La figura 3 muestra que en el cantón de Matina existen 11 áreas macroambientales según las variables utilizadas en este estudio. Todas las áreas fueron comunes a ambos años con excepción de la 15 y la 16 que solo están demarcadas en el 2005.

Figura 3. Dendrograma mostrando las características de los grupos macroambientales en el cantón de Matina.

\section{Clasificación de áreas macroambientales.}

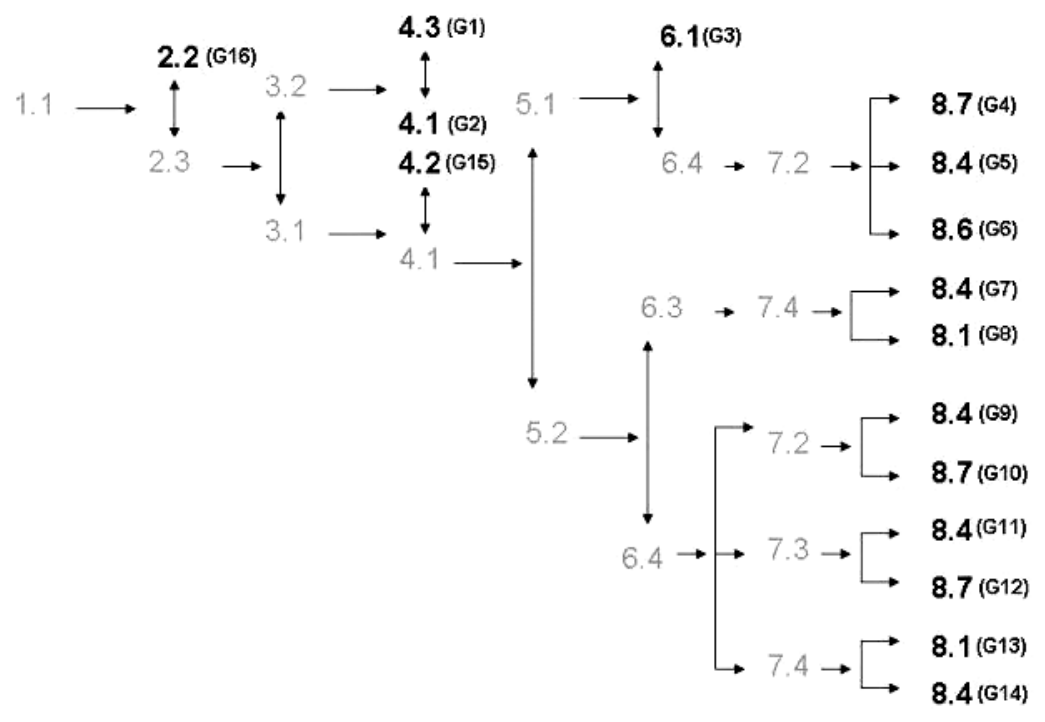


Figura 4. Distribución de las áreas macroambientales del cantón de Matina indicando aquellas del área de referencia de Estrada.

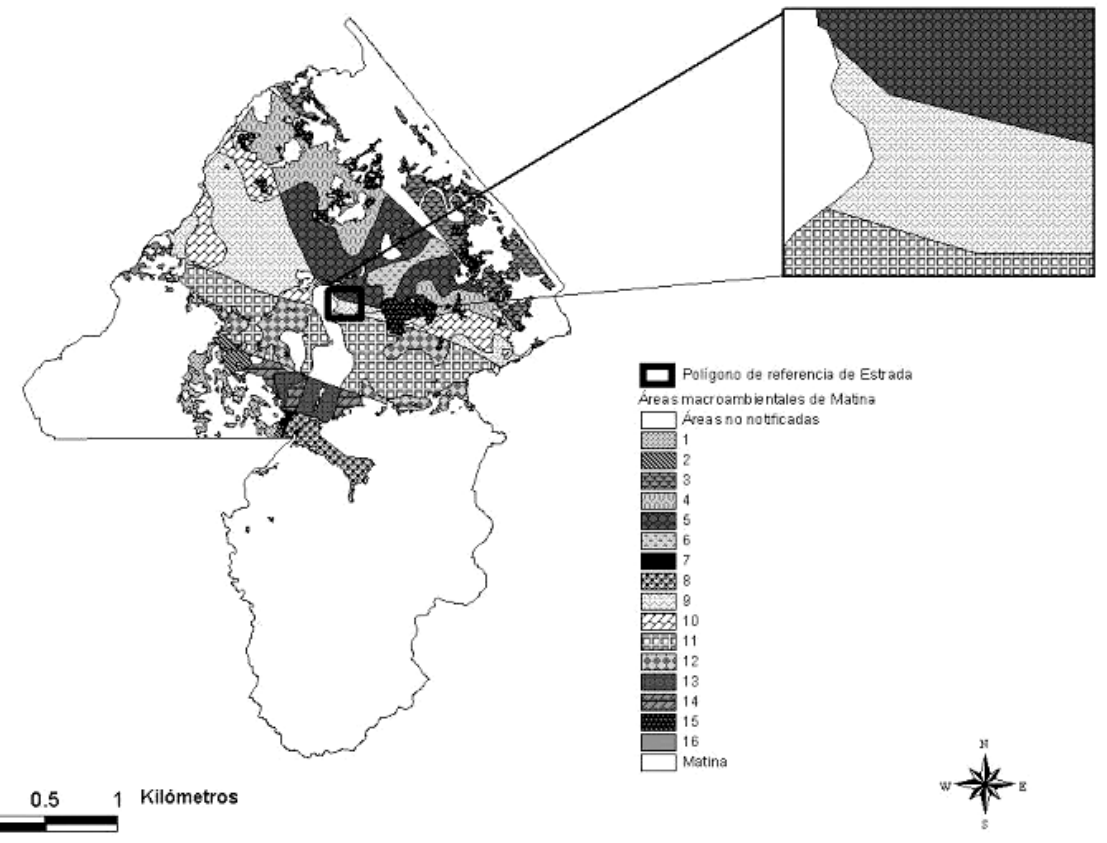

Los cuadros 2 y 3, muestran las variables cuantitativas y cualitativas, generadas mediante la encuesta de vivienda, que son, significativamente, diferentes entre el grupo positivo y el negativo. Todas se relacionan con la población que habita en las casas, el tiempo que han vivido allí, tenencia de la propiedad, la estructura de la vivienda y otras características de la vivienda. 
Cuadro 2. Mediana, valor mínimo, valor máximo y rango para las características cuantitativas de las viviendas de Estrada, significativamente, diferentes entre casas positivas y negativas.

\begin{tabular}{|c|c|c|c|c|c|c|c|c|c|}
\hline \multirow[b]{3}{*}{ Variable estudiada } & \multicolumn{8}{|c|}{ Resultado obtenido } & \multirow{3}{*}{$\begin{array}{c}\text { Valor de } p \\
(2 \text { colas })\end{array}$} \\
\hline & \multicolumn{4}{|c|}{ Positivos } & \multicolumn{4}{|c|}{ Negativos } & \\
\hline & Mediana & Min & Max & Rango & Mediana & Min & Max & Rango & \\
\hline \multicolumn{10}{|l|}{$\begin{array}{l}\text { Población que habita } \\
\text { la casa }\end{array}$} \\
\hline $\begin{array}{l}\text { Número total de } \\
\text { miembros de la familia }\end{array}$ & 4 & 1 & 13 & 12 & 4 & 1 & 10 & 9 & 0.000123 \\
\hline Número de hombres & 2 & 0 & 7 & 7 & 2 & 0 & 6 & 6 & $5.2 \times 10^{-5}$ \\
\hline $\begin{array}{l}\text { Número de menores } \\
\text { de } 18 \text { años }\end{array}$ & 2 & 0 & 8 & 8 & 2 & 0 & 6 & 6 & 0.0175 \\
\hline \multicolumn{10}{|l|}{$\begin{array}{l}\text { Características de las } \\
\text { viviendas }\end{array}$} \\
\hline $\begin{array}{l}\text { Número de días } \\
\text { habitados por la } \\
\text { familia actual }\end{array}$ & 1825 & 4 & 14600 & 14596 & 1460 & 1 & 27740 & 27739 & 0.004 \\
\hline
\end{tabular}

Cuadro 3. Frecuencia observada, valor de p y riesgo relativo de las características cualitativas de las viviendas de Estrada que son, significativamente, diferentes entre casas positivas y negativas.

\begin{tabular}{|c|c|c|c|c|c|}
\hline \multirow[b]{2}{*}{ Variable estudiada } & \multirow[b]{2}{*}{ Categoría } & \multicolumn{2}{|c|}{ Resultado obtenido } & \multirow[b]{2}{*}{$\begin{array}{l}\text { Valor } \\
\text { de p }\end{array}$} & \multirow[b]{2}{*}{$\mathbf{R R}$} \\
\hline & & $\begin{array}{c}\text { Positivos } \\
\text { (frecuencia) }\end{array}$ & $\begin{array}{c}\text { Negativos } \\
\text { (frecuencia) }\end{array}$ & & \\
\hline \multicolumn{6}{|l|}{ Estado socioeconómico de la familia } \\
\hline Tenencia de vivienda & Propia & 0.61 & 0.49 & 0.0270 & 1.25 \\
\hline \multicolumn{6}{|l|}{ Estructura de la vivienda } \\
\hline Materiales de las paredes & Cartón & 0.02 & 0.07 & 0.0415 & 0.31 \\
\hline \multicolumn{6}{|l|}{ Otras características de la vivienda } \\
\hline $\begin{array}{l}\text { Tipo de cocina principal por fuente } \\
\text { de energía }\end{array}$ & No tiene & 0.02 & 0.10 & 0.0059 & 0.22 \\
\hline \multirow[t]{2}{*}{ Acceso a electricidad } & Sí & 0.98 & 0.93 & 0.0149 & 1.06 \\
\hline & No & 0.01 & 0.07 & 0.0149 & 0.20 \\
\hline \multirow[t]{2}{*}{ Abastecimiento de agua } & Cañería & 0.83 & 0.93 & 0.0037 & 0.89 \\
\hline & Pozo con bomba & 0.04 & 0 & 0.0059 & - \\
\hline \multirow[t]{2}{*}{ Vivienda con pozo artesanal } & Sí & 0.14 & 0.07 & 0.0328 & 2.06 \\
\hline & No & 0.86 & 0.93 & 0.0328 & 0.92 \\
\hline \multirow[t]{2}{*}{ Estado de la iluminación } & Buena & 0.65 & 0.52 & 0.0121 & 1.26 \\
\hline & Regular & 0.30 & 0.44 & 0.0106 & 0.69 \\
\hline Tipo de baño & Colectivo & 0.15 & 0.25 & 0.0277 & 0.60 \\
\hline Ubicación del baño & No tiene & 0.02 & 0 & 0.031 & - \\
\hline
\end{tabular}


La figura 5, muestra la distribución del IPA en el cantón de Matina, como puede apreciarse la zona donde es mayor el IPA corresponde con las áreas macroambientales 5 y 10. El RR y el \% RAP para estas áreas se muestran en el cuadro 4.

Figura 5. Distribución del promedio del IPA (Incidencia Parasitaria Anual) entre años.
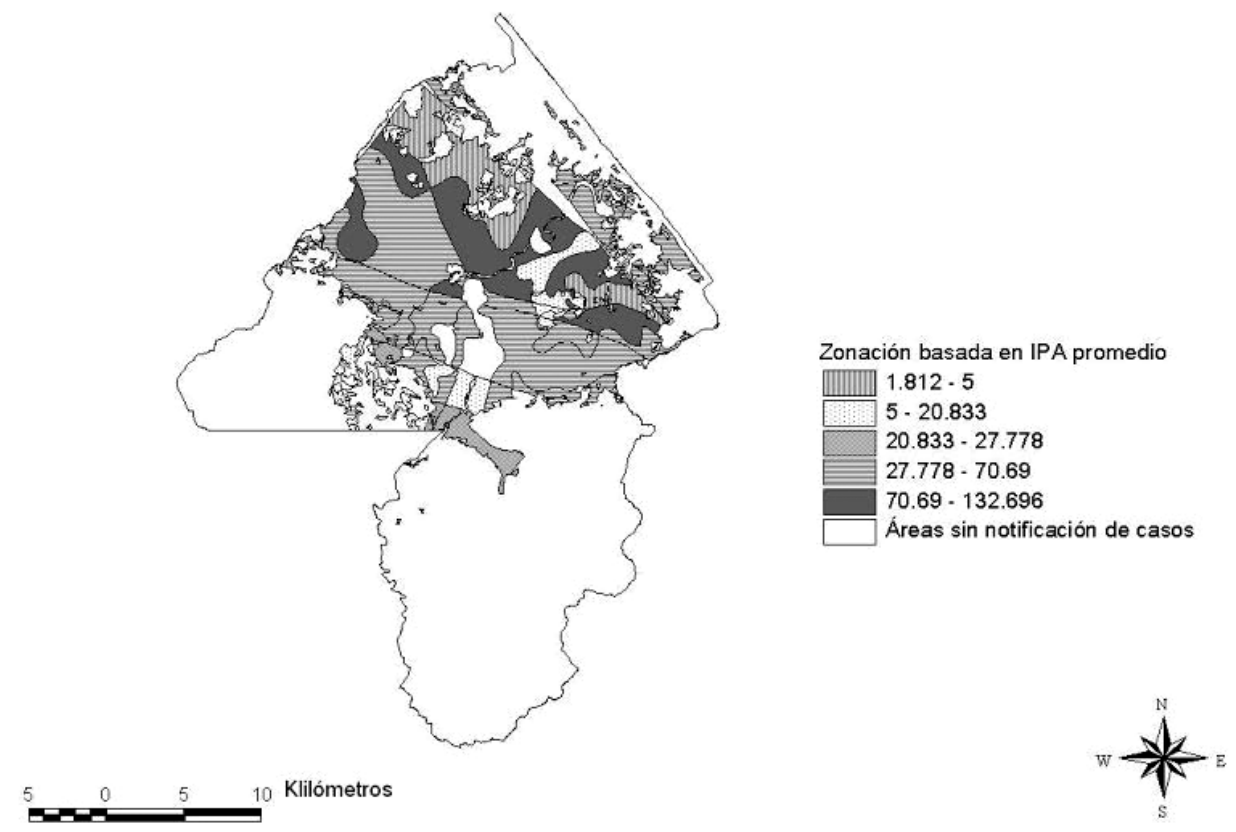

Cuadro 4. IPA, RR y \% RAP en cada una de las áreas macroambientales en Matina para las áreas con un riesgo relativo $>1$.

\begin{tabular}{|c|c|c|c|c|}
\hline \multirow{2}{*}{ Área } & Año & IPA & RR & \%RAP \\
\hline \multirow{2}{*}{5} & 2005 & 148.8514 & 2.6145 & 27.90 \\
\cline { 2 - 5 } & 2006 & 116.5414 & 1.7402 & 15.20 \\
\hline \multirow{2}{*}{9} & 2005 & 78.1630 & 1.0000 & 0 \\
\cline { 2 - 5 } & 2006 & 67.2162 & 1.0307 & 0.79 \\
\hline \multirow{2}{*}{10} & 2005 & 133.9255 & 1.7922 & 1.66 \\
\cline { 2 - 5 } & 2006 & 90.0570 & 1.3967 & 0.85 \\
\hline
\end{tabular}


Las Figuras 6 y 7, muestran, respectivamente, la distribución temporal de la actividad malárica por localidad y por vivienda en la localidad de Estrada. A nivel de localidad, a pesar de que la serie temporal es pequeña, se observa que la actividad es continua y fluctuante durante todo el año, no llegando nunca a cero. Como resultado del ANDEVA no existen diferencias significativas entre meses en cuanto a la latitud y la longitud, siendo la probabilidad 0.9994 para la primera y 0.9999 para la segunda.

Figura 6. Presentación temporal del número de casos por localidad y el número de localidades, por mes, durante el periodo de observación (2005-2006)
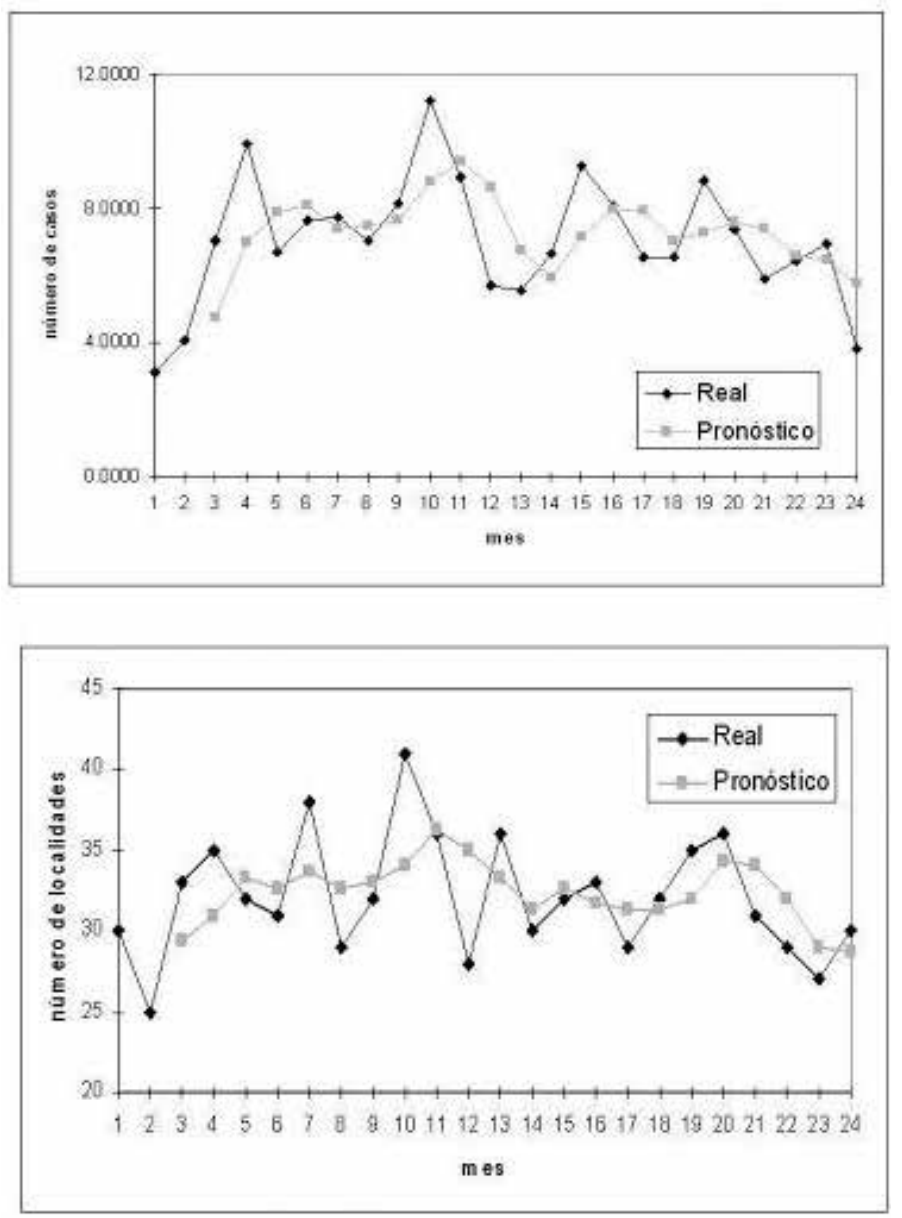
Figura 7. Presentación temporal del número de casos y el número de viviendas, por mes, durante el periodo de observación (2005-2006)
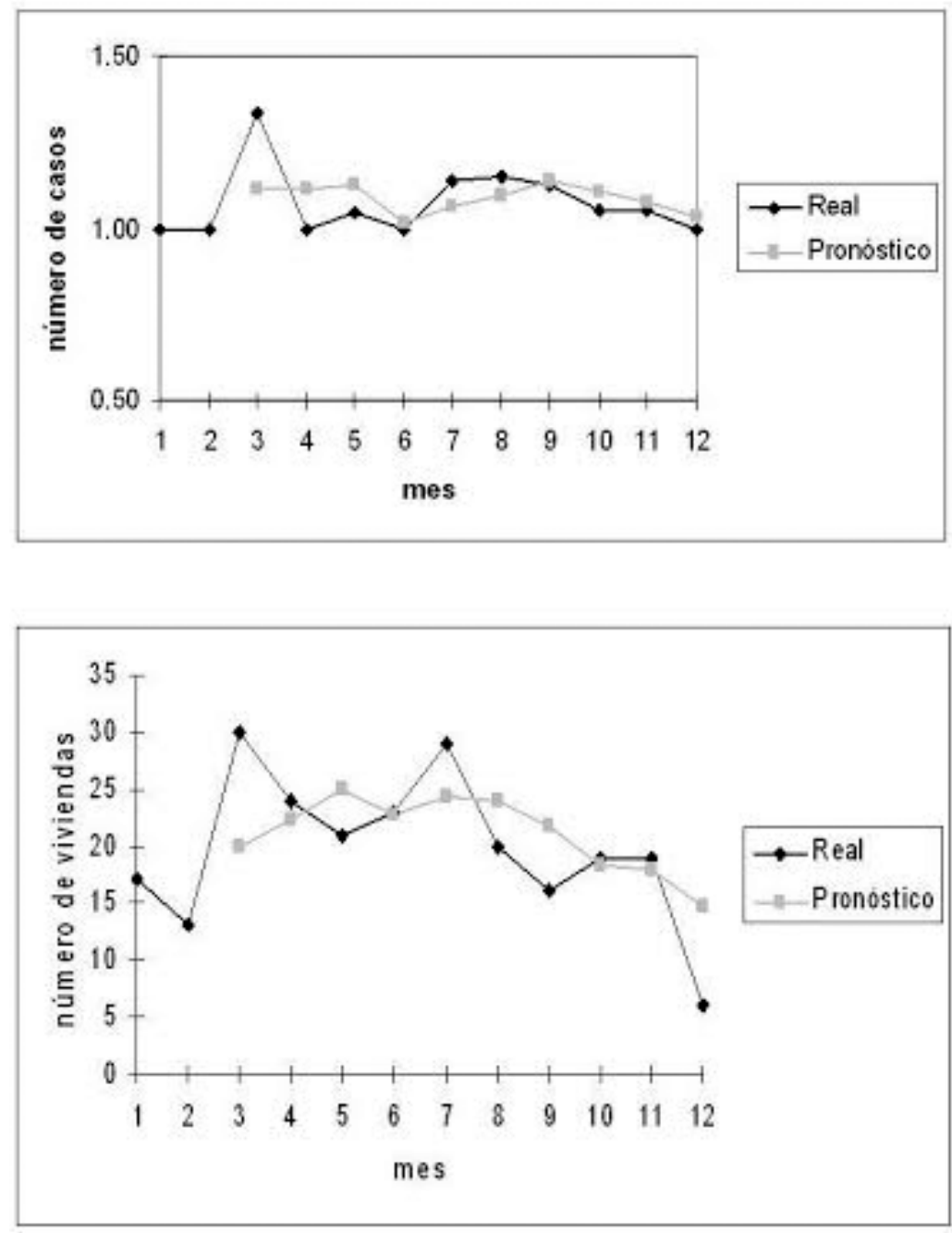

Esto concuerda con la distribución concéntrica de los centros promedio por mes y la poca fluctuación de los promedios de las coordenadas por mes. El rango de confianza al 95\% del promedio de los promedios para la longitud fue de 614033.7760 a 614438.841 y para la latitud fue de 227043.8584 a 227348.8878 . El coeficiente de variación fue de $0.08 \%$ y $0.16 \%$ respectivamente. 
A nivel de vivienda tampoco se encontraron diferencias significativas entre la longitud $(p=0.9952)$ y la latitud $(p=0.1625)$ y se observó poca oscilación mensual en estas variables. El intervalo de confianza de 95\% para la longitud fue de 615284.057 a 615326.737 y para la latitud de 227106.3163 a 227320.0563 . El coeficiente de variación fue menor en la longitud $(0.005 \%)$ que en la latitud $(0.074 \%)$.

Todas las localidades del cantón de Matina están a una distancia menor de $2 \mathrm{~km}$ de un cuerpo de agua (río) que podría ser criadero potencial del vector.

\section{Discusión de resultados}

La vigilancia activa para la detección temprana de los casos de malaria, se logra gracias a los técnicos de atención primaria de la salud de la CCSS y del Ministerio de Salud, a los colaboradores voluntarios y a la cooperación comunitaria. La toma de información y el seguimiento apropiado en cada caso se requiere para garantizar el control adecuado de la enfermedad mediante el tratamiento temprano.

El manejo adecuado de la información generada en este proceso podría ser facilitado mediante el uso de un sistema georeferenciado de manejo de la información. Los sistemas de información geográfica son una herramienta para recuperar, almacenar, analizar y desplegar la información. Tienen la ventaja de ser actualizados en forma continua y de identificar en cualquier momento el origen geográfico de la información digitada. En el caso de la malaria, la identificación precisa de las áreas de alto riesgo es imprescindible para el éxito de los programas de control (Nath et al., 2013). Muchos otros eventos de salud necesitan poder ser manejados espacialmente para controlarlos eficiente y eficazmente, entre estos, la incidencia de enfermedades infecciosas en localidades rurales.

Las bases de datos relacionales creadas con el SIG, permiten la adición, modificación o eliminación de campos o registros en forma continua, de tal manera que su actualización puede ser inmediata. En un estudio de situación, para obtener información actualizada o para monitorear los cambios ocurridos en el tiempo, este instrumento es útil para el proceso de toma de decisiones. Estudios anteriores han utilizado los SIG para mapear y evaluar la malaria en distintos niveles geográficos (Fuller et al., 2014, Ngom y Siegmund 2014; Nath et al., 2013, Hanafi-Bojd et al., 2012, Zayeri, Salehi y Pirhsseini, 2011, Lek-Uthai et al., 2010, Alegana et al., 2009). 
La información presentada aquí debe considerarse entonces como una línea de base para los estudios futuros en la misma región y no como un producto terminado per se.

En el caso particular de Matina no fue posible determinar la existencia de localidades en las que no se hubieran encontrado casos de malaria en el 2005 o en el 2006; sin embargo, sí se observaron diferencias en el número de casos informados en cada localidad. La distribución de la enfermedad en Matina fue similar entre años, 2005 y 2006, como se indica por la no existencia de diferencias significativas en el centro promedio. Cuatro localidades difirieron, 2 presentándose, únicamente, en el 2005 y 2, solamente en el 2006.

Previos sistemas de clasificación ambiental, tales como el sistema de zonas de vida de Holdridge (Tosi, 1969) o el sistema de unidades bióticas de Herrera y Gómez (Herrera y Gómez, 1993), indican que el cantón de Matina es bastante homogéneo en sus características de clima. Los mapas producidos utilizando estos sistemas tienen una escala de 1:750 $000 \mathrm{y}$ 1:685 000, respectivamente.

En el caso del sistema Holdridge las áreas se clasifican como Bosque Húmedo Tropical (bh-T) o Bosque Muy Húmedo Premontano (bmh-T). El primero, caracterizado por pertenecer a la provincia de humedad húmedo; una relación de evapotranspiración potencial de 0.50 a 1.00 , un promedio de de precipitación por año en milímetros entre $2000 \mathrm{~mm}$ y $4000 \mathrm{~mm}$ y una biotemperatura media anual de $>24^{\circ} \mathrm{C}$. Y el segundo, caracterizado por pertenecer a la provincia de humedad perhúmedo, relación de evapotranspiración potencial de 0.25 a 0.50 , promedio de precipitación por año en milímetros entre 4000 $\mathrm{mm}$ y $8000 \mathrm{~mm}$ y, biotemperatura media anual $>24^{\circ} \mathrm{C}$.

En el sistema de Herrera y Gómez la zona comprendida dentro del cantón de Matina, se considera como T/T-h/0 (Tropical, Tropical húmeda, sin meses secos) o T/T-mh/0 (Tropical, tropical muy húmeda, sin meses secos). Para ambos casos sobresale la ausencia de meses secos.

Para el propósito de este estudio, se consideró proponer otro sistema de clasificación que incluyera además de las variables contempladas en sistemas previos (elevación, meses secos, precipitación) otras sobre la naturaleza, características y uso del suelo (geología, características del terreno, tipo de suelo, uso actual del suelo) y brillo solar. La racionalidad de esta decisión es que la malaria es una enfermedad afectada por diversos 
factores ambientales (Hanafi-Bojd et al., 2012), esto debido a que el vector de la enfermedad se reproduce en aguas estancadas a consecuencia de las propiedades del terreno. En el estudio conducido por Lek-Uthai y colaboradores (2010), la mayoría de los casos estuvieron localizados cerca de cuerpos de agua, lo que sugiere que el ambiente y los factores entomológicos juegan un papel importante en la incidencia de la malaria.

Todas las capas utilizadas para esta caracterización tenían una escala menor o igual a 1:200 000 lo que implica un menor nivel de agregación de los datos. Aún así, las escalas utilizadas y el uso de capas con distintas escalas se convierten en una limitante para el estudio ya que se podrían dejar de lado detalles que tal vez se estén relacionados con la distribución del vector y la incidencia de la enfermedad (Fuller et al., 2014).

Las diferencias en la distribución del IPA podrían ser explicadas por factores ambientales (macro o micro). En este caso se propone un sistema de clasificación de áreas basado en 8 características. Los grupos identificados en el 2005 y en el 2006 correspondieron a las mismas áreas con excepción de las áreas 15 y 16 que solo se presentaron en el 2005. Durante este estudio fue posible determinar que las áreas 5, 9 y 10 tienen un riesgo relativo mayor para la ocurrencia de malaria.

El \%RAP fue bajo para las tres áreas anteriormente mencionadas, siendo el más alto el del área 5 que es para cada uno de los dos años $<30 \%$, lo cual pareciera indicar que otros factores no detectados en este estudio están actuando como determinantes de la enfermedad. Factores de índole social no fueron suficientemente explorados durante este estudio.

La actividad malárica en el cantón de Matina durante el año es continua, como es indicado por la fluctuación en el número de localidades positivas y el número de casos por mes. Lo anterior pareciera sugerir que en todo momento hay contacto entre vector y huésped lo que incita a proponer un estudio de estacionalidad del A. albimanus en la zona o una búsqueda activa de vectores secundarios en el área.

A nivel de la localidad piloto, a pesar de hallarse ubicada en tres áreas macroambientales, no se observaron diferencias en la incidencia de la enfermedad entre áreas. Las viviendas positivas y negativas son concéntricas no habiendo diferencias significativas en su centro promedio. La actividad malárica es continua durante el año, como lo indica la ocurrencia 
de casas con al menos un caso reportado durante cada uno de los 24 meses del estudio.

Tomando en cuenta los resultados del análisis temporal de los centros promedios por mes, se puede concluir que el evento de la enfermedad ocurre siempre en la misma zona, tanto a nivel del cantón como en la localidad piloto, lo cual debería de conducir los esfuerzos de las entidades de salud hacia ella. Los coeficientes de variación de la longitud y de la latitud sugieren que existe mayor dispersión en la latitud.

A nivel microambiental, existen pocos factores que se pudieron identificar como de riesgo, entre estos, aquellos relacionados con los habitantes y con la vivienda y su estructura. A pesar de existir el concepto de casa malárica, los únicos factores identificados se relacionaban con el tiempo de vivir en la casa, la tenencia de la propiedad, el acceso a la electricidad, el uso de pozo artesanal y el estado de iluminación. Estos factores podrían estar asociados con un estatus socioeconómico, resultados de otros estudios han demostrado que la ocurrencia de la malaria está relacionada con el estatus socioeconómico (Ngom y Siegmund, 2014).

Podrían realizarse estudios futuros sobre los vectores de la enfermedad en la zona y sus hábitos de vida, que los relacionan con los humanos en un medio principalmente agrícola, dedicado en su mayor parte al monocultivo extensivo. Es claro que el área tiene una alta precipitación durante todo el año, un sistema complejo de irrigación natural (ríos), un sistema elaborado de irrigación artificial representado por la red de canales en las plantaciones bananeras y una alta temperatura durante todo el año. Otros estudios concuerdan que las áreas cultivas son los criaderos más importantes para el vector de la malaria (Ngom y Siegmund, 2014). No cabe duda que las actividades antropogénicas deberán ser evaluadas para comprender su impacto en el mantenimiento del ciclo de transmisión de la enfermedad.

Estos factores entomológicos y socioculturales necesitan ser estudiados para comprender el padrón espacial descrito en este trabajo.

Cambios en el patrón espacial actual son esperables debido a las intervenciones para el control de la enfermedad, las actividades antropogénicas en la zona y los cambios climáticos; tener una línea de base en formato electrónico permite dar seguimiento a los cambios observados, en particular, si se logra la transferencia de las nuevas tecnologías espaciales a los grupos de campo encargados de la colecta primaria de la información. 


\section{Agradecimientos y colaboradores}

Al personal del Área Rectora del Ministerio de Salud en Matina en la provincia de Limón. Al Dr. Gerardo Galvis de la Organización Panamericana de la Salud (OPS) y al Dr. José Luís Garcés de la unidad de Vigilancia de la Salud del Ministerio de Salud a nivel central. Al "Programa Regional de Acción y Demostración de Alternativas Sostenibles para el Control de los Vectores de la Malaria Sin Uso del DDT en México y América Central" (Proyecto DDT/GEF ) de donde se obtuvieron los fondos para la ejecución de este trabajo.

\section{Referencias}

Alegana, V., Atkinson, P., Wright, J., Kamwi, R., Uusiku, P., Katokele. S., Snow, R. y Noor, A. (2009). Estimation of malaria incidence in northen Namibia in 2009 using Bayesian conditional-autoregressive spatial-temporal models. Spatial and Spatio-temporal Epidemiolo$g y, 7,25-36$.

Fuller, D.O., Troyo, A., Alimi, T.O. y Beier, J.C. (2014). Participatory risk mapping of malaria vector exposure in northern South America using environmental and population data. Applied Geography, 48, $1-7$.

Hanafi-Bojd, A.A., Vatandoost, H., Oshaghi, M.A., Charrahy, Z., Haghdoost, A.A., Zamani, G., Abedi, F., Sedaghat, M.M., Soltani, M., Shahi, M. y Raeisi, A. (2012). Acta Tropica, 122,132-137.

Herrera-Soto, W. y Gómez-Pignataro, LD. 1993. Mapa de Unidades Bióticas de Costa Rica.

Lek-Uthai, U., Sangsayan, J., Kachenchart, B., Kulpradit, K., Sujirarat, D. y Honda, K. (2010). Novel ellipsoid spatial analysis for determining malaria risk at the village level. Acta Tropica, 116, 51-60.

Nath, M.J., Bora, A.K., Yadav, K., Talukdar, P.K., Dhiman, S., Baruah, I. y Singh, L. (2013). Prioritizing areas for malaria control using geographical information system in Sonitpur district, Assam, India. Public Health, 127 572-578.

Ngom, R. y Siegmund, A. (2014). The key role of socio-demographic and socio-environmental factors in urban malaria occurrance and control- An illustration using the city of Yaoundé. Social Science and Medicine $x x x, 1-11$. 
Ortiz, E. (2004). Atlas digital de Costa Rica. Versión 2004. Instituto Tecnológico de Costa Rica.

Tosi, AJ. (1969). Mapa Ecológico de Costa Rica basado en zonas de vida. Centro Científico Tropical. San José. Costa Rica.

Zayeri, F., Salehi, M. y Pirhsseini, H. (2011). Geographical mapping and Bayesian spatial modeling of malaria incidence in Sistan and Baluchistan province, Iran. Asian Pacific Journal of Tropical Medicine xxx, 985-992. 